\title{
Open Detector of Alpha Particles based on GEM-like Structure
}

\author{
V. I. Razin \\ Institute for Nuclear Researches, Russian Academy of Sciences, Russia
}

Copyright (C) 2015 by authors, all rights reserved. Authors agree that this article remains permanently open access under the terms of the Creative Commons Attribution License 4.0 International License

\begin{abstract}
This paper presents the results of studies of the characteristics of alpha particles detector like TGEM ("Thick" Gas Electron Multiplier) working in an open air environment. The main advantages of these devices are simplicity, low cost, and high sensitivity to the different kinds of irradiation. One of the possible applications of these new wireless gas detectors is alpha particle or radon background monitors, which can serve also as a dosimeter or as a smoke detector in houses. This detector is able to measure the trace of alpha particles with efficiency close to $100 \%$ operating in open air in the avalanche mode at high gas gain (up $10^{4}$ ). The tests were carried out in duty of three summer months. It should be noted that the stability of the results is high enough despite the wide range of temperature and humidity during of testing.
\end{abstract}

Keywords Gas Detector, Electron Multiplication, Gas Electron Multiplier, Resistive Electrode, GEM TGEM, RETGEM

\section{Introduction}

In experimental nuclear physics two ways of registration of ionizing radiation by mean of air gas detectors most are widely adopted. Along with integrated ionization chambers, which measure a total ionization for a certain period, there is other class of the so-called pulse ionization chambers intended for registration of impulses of current when passing via detector of separate ionizing particles [1-4].The design both types of the chambers differs from each other a little. Distinction consists only in electronic methods of reading of size of ionization current. Thus the full duration of the induced signal including fast electronic and slow ionic components is depending on geometry of electrodes and a nature of the filling gas. According to experimental data [5] drift velocity of electron in the mainly of the used gases is equal about $10^{6} \mathrm{~cm} / \mathrm{sec}$ in the range of E/P from 1 to $20 \mathrm{~V}$ $/ \mathrm{cm}$. Hg. The working voltage gets out such that the processes of recombination and diffusion of electrons had the minimum size and did not influence on the value of the efficiency of collecting a charge. The detector with air has the high probability of formation of negative ions. In this case the forward front of induce pulse will have the same duration as well as the back front caused by the movement of positive ions. As a result high-speed ability of the detector with air filling at least 100 times concedes to similar devices in which the probability of formation of negative ions has the small value. It means that air ionization chambers or open chambers allow to measure ionization without electron multiplication and to test the detectors of this type by means of radioactive sources with the minimum dark current at the level of $10 \mathrm{pA}$. Detectors of this type find application as in the medical purposes at radiation therapy with use of different types of the ionizing particles, and for scientific experiments in the field of physics of nuclear and elementary particles at measurement of coordinates and losses of energy with an energy threshold up to $0.3 \mathrm{MeV} /$ nucleon [6].

\section{The Open Air Detector Working with Gas Amplification}

The open or without walls detectors operating at air with electron multiplication have considerable advantage in comparison with the gas proportional counters or Geiger-Muller counters[7-8].The open counter is the unique device for the account in one run of the low energy electrons or beta radiation, and also alpha particles , $\mathrm{x}$-ray and gamma radiations. In the open detector electrons move as negative ions of oxygen towards positively charge anode. At the according potential difference between electrodes it is possible to reach such intensity of electrical field at which a separation of an electrons and its avalanche reproduction are occurred. In this case dead time of the detector will be defined by a sum of duration of ion drift time until a separation of an electron and time of interrupting of discharge by means of electrical chain. The number of negative ions coming to area of electron avalanche 
multiplication is depending on number of impacts with air gas molecules. Therefore the velocity of the account of the charged particles registered by the detector is established in a wide interval from 1 to a maximum depending on a type of radiation and high voltage between electrodes. In this case the high speed ability of the detector of this type considerably surpasses values which are characteristic for the ionization chambers discussed above. It should be note also that efficiency of registration, for example, alpha particles on air in the avalanche mode at the high level of coefficient of gas multiplication can reach $100 \%$ value independently from of air humidity [9-11].

The main advantage of the gas detectors operating in the pulse mode at air is defined by their simplicity, low cost and high sensitivity to single events. One of possible applications this type of devices is monitoring of the background from traces of alpha particles. Owing to low manufacturing cost and simplicity of using they can be served as sensors of radon both in the closed office rooms and in the open spaces where are many people: airports, railway stations, forest and etc.

This work has the purpose to continue research of possibility of operation of the gas detector filled with air both in current and in pulse modes with gas amplification taking into account the last achievements in the field of development of gas multiplier like GEM [12]

\section{The Open GEM-like Detector}

According to the data representative above it is real to consider that efficiency of the registration of trace of alpha particles or radon in environment air significantly increases when using the gas multiplication mode for improve of the relation a signal-noise. At the same time it should be noted that operation of such detector does not differ in high stability because of spontaneous transition of the avalanche mode to corona discharge by means the mechanism of the back positive ion and photon connections [13-16]. Further research of the way of improvement of the characteristics of this class of detectors with air filling led to encourage results in due to the emergence of a new type of hole position sensitive devices, and also resistive gas electron multipliers [17-18].As a results of tests of this type detectors it has been found that for every percentage of water in the environment this hole or highly resistive electrodes structures has sufficient sensitivity at registration of the trace of alpha particles or radon. Leakage currents between the resistive hole electrodes were kept to a minimum (about $10 \mathrm{pA}$ ) through the use of dielectric spacers located in dielectric areas of electrodes. Photon feedback has been almost suppressed by the geometric features of the hole electrode structure. However the process of manufacturing of the resistive electrodes includes etching microscopic rims around the holes. Deposition of a resistive layer and other complex chemical processes are some difficulties that can limit a mass production and using of such structures.
Fig. 1 shows the hole detector developed by the author taking into account the advantages and disadvantages of the previous devices of this type [19]. The main difference of this design is used as hole electrode thin metal mesh made of brass, widely applied in electronic industry. By means of well-developed technology of etching holes without burrs can minimize the breakdown phenomena due to mechanical defects. The body 1 of the detector provides for place a source of alpha particles on the substrate 2. Distance to positive electrode 3 can be changed in the range of 2-10 $\mathrm{mm}$. in accordance with the task of experiment. The gap between electrodes 3 and 4 is adjusting by mean of dielectric spacers 5 , placed in isolated areas of the hole electrodes to prevent the large leakage current. High voltage was applied to the negative electrode 4. Signals from both electrodes are reading by dividing high-voltage capacitors to high-impedance input of the oscilloscope.

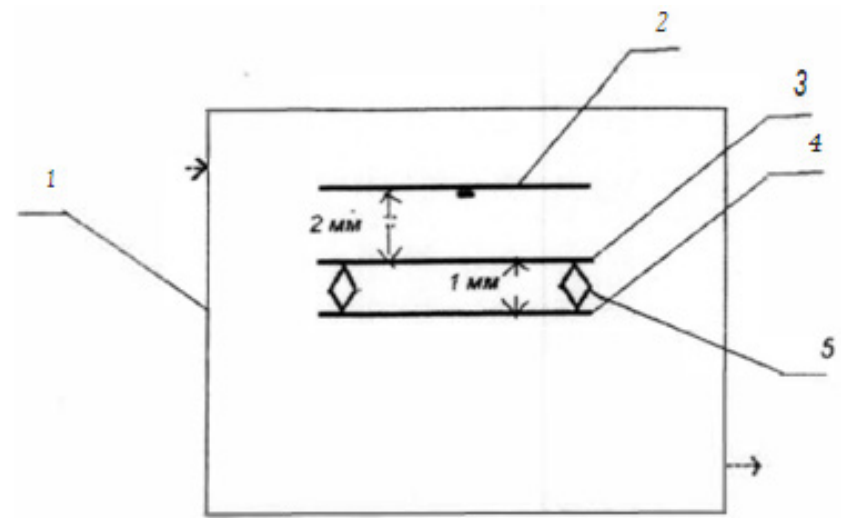

Figure 1. A schematic view of the open detector 1-body with inlet and outlet for the air ог gas mixture; 2-plate with alpha partic/es ; 3-greed-anode; 4-greed-cathode

Fig.2 shows the geometric dimensions of the elements of one of the sample brass mesh selected as hole electrode. From point of view of the optimum geometry of GEM the sample is accepted with the following parameters:

$\begin{array}{lc}\text { Electrode thickness } & 50 \mu \mathrm{m}, \\ \text { Hole geometry } & \text { square of } \\ & 400 * 400 \mu \mathrm{m}, \\ \text { Humidity of air } & 80 \%, \\ \text { Atmosphere pressure } & 754 \mathrm{~mm} . \mathrm{Hg} .\end{array}$

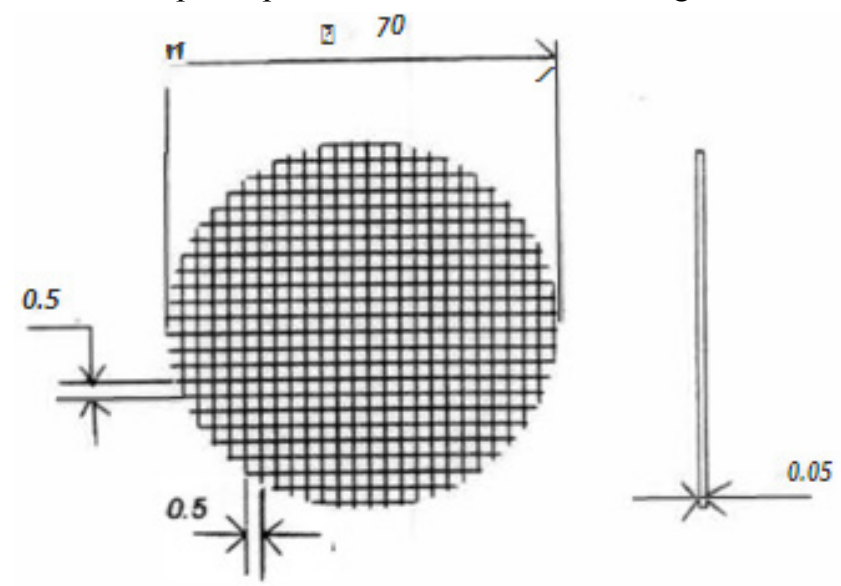

Figure 2. The geometric dimensions of the metal greed 


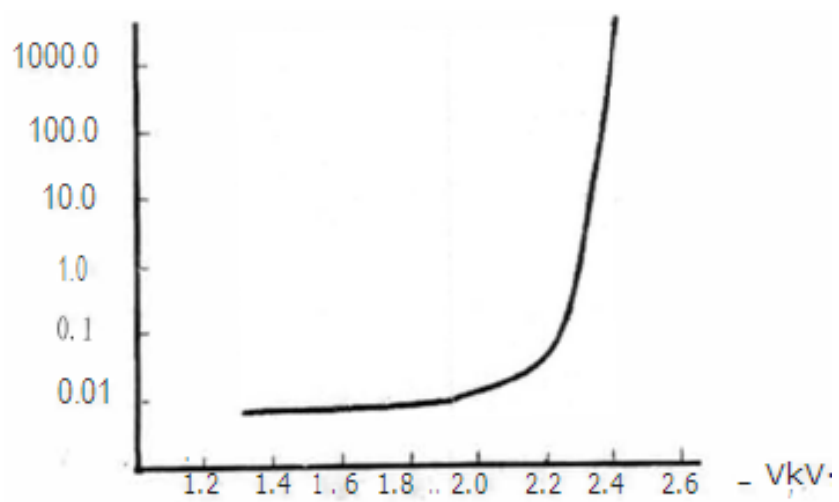

Figure 3. The current-voltage characteristic of the detector type GEM in air

Fig. 3 shows the current-voltage characteristic of the test detector with holes electrodes in the air. The size of the gap between electrodes is $1 \mathrm{~mm}$.

These measurements were performed in order to verify the assembly technology as well as detecting and eliminating points of sparks in the absence of irradiation.

Fig.4 presents the amplitude characteristics of the hole detector like GEM at radiation by an alpha source of $\mathrm{Ra}^{226}$ with intensity about $10^{4}$ particles a second in the open air with the gas mixtures $\mathrm{Ar}+20 \% \mathrm{CO}_{2}, \mathrm{Ar}+10 \% \mathrm{CH}_{4}+4 \% \mathrm{SF}_{6}$.

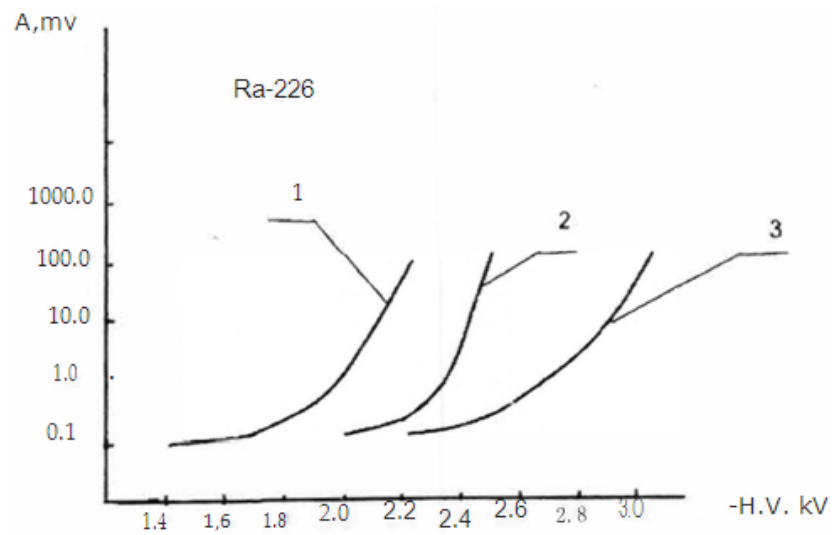

Figure 4. Amplitude characteristics of the detector type GEM: 1-in amblent air; 2-with $\mathrm{Ar}+20 \% \mathrm{CO}_{2}$ gas mixture; 3-with $\mathrm{Ar}+10 \% \mathrm{CH}_{4}+4 \% \mathrm{SF} 6$ mixture

5. Fig.5 shows the dependence of the amplitude of the pulse vs time irradiation by alpha source with low intensity. Of course curve shows that the pulse amplitude varies slightly at a fairly significant fluctuation in temperature and humidity in the open room.

\section{Discussion of Results}

In this paper one of ways of creation of alpha particles detectors operating in air environment with efficiency near to $100 \%$ of it and other types of radiation is shown. This possibility appeared as a result of improvement of the relation a signal-noise due to application of new type of the open devices which stability worked in the electron avalanche mode. This stability is reached in the hole gas electron multiplier (GEM).In this case the photon feedback, causing the spontaneous transition from the avalanche mode to corona discharge is eliminated. Thus there is a real possibility to measure the trace of alpha particles with energy about $60 \mathrm{keV}$ not only in gas or air volumes but to make a scanning of a surface of the irradiation subjects as length of the free run of alpha particles on air makes not less of $4 \mathrm{~cm}$.

It should also take into account the fact that a probability of recombination of negative and positive ions is negligible during the drift of negative ions in the gap with a strong electric field. Together with the appearance of free electrons resulting from remove of the electron from the negative ions in strong electrical field these processes can be a cause of growth signal to noise ratio. These results may serve as sufficient arguments to measure the radioactive traces any type with open air GEM-like detectors.

\section{Conclusions}

The proposed design of an open air GEM like detector provides measurement of traces of alpha particles and other types of radiation with an efficiency close to $100 \%$. Due to its low cost to manufacture as well as a stable operation for a long time of the irradiation (at least 6 months), this device can be widely used as a monitor of radioactive contamination in the environment over a wide range of applications.

\section{REFERENCES}

[1] E.Marode, The mechanism of spark breakdown in air at atmospheric pressure between a positive point and a plane. Experimental: Nature of the streamer track, Journal of Applied Phys., Vol.46,№5, May 1975.

[2] S. I. Potashev, S.V.Akulinichev,Ju.M.Burmistrov et al., A thin wall multichannel air ionization chamber, Instr. and Exper. Tech., 47(4), 2004,516

[3] T.Noguchi,S.Nagashima,M.Uda. An electron counting mechanism for the open counter operated in air, Nucl.Instr.and Meth.A 342(1994),521-526

[4] L.Buermann,G.Hilgers, Significant discrepancies in air kerma rates measured with free-air and cavity ionization chambers, Nucl. Instr.and Meth.A580, 2007,477.

[5] V.I.Razin, The choice of the optimum gas mixture for high rate wire chambers, Nucl.Instr.and Meth.A 367(1995), 295-297.

[6] G. Charpak, P. Benaben, P. Breuil, V.Peskov, Detector of alpha particles and X-rays operating in pulse counting mode or/and gas amplification,2008,JINST,3 ,PO 2006.

[7] F. Sauli, GEM: A new concept for electron multiplication in gas detector, Nucl.Instr. and Meth. A 386 (1997), pp.531-534.

[8] G. Charpak et al., Development of New Hole-Type Detectors and the first results of Their Applications, IEEE 
Trans.Nucl.Sci. 55, (2008), 1657.

[9] G. Charpak, P.Benaben, P.Breuli, P.Martinengo, E.Nappi and V.Peskov, Progress in the development of a S-RETGEM-based detector for early forest warning system,2009,JINST,4P 12007.

[10] J.Ostling et al., Study of Hole-Type Gas Multiplication Structures for Portal Imaging and Other High Count Rate Applications, IEEE Trans.Nucl.Sci.50(2003),809.

[11] J.M. Bidault et al., The first applications of newly developed gaseous detectors with resistive electrodes for UV imaging in daylight conditions, Nucl.Instr. and Meth. A 580(2007), 1036.

[12] V.Peskov et al., Development and first Tests of GEM-like detectors with Resistive Electrodes, IEEE Trans.Nucl.Sci.Vol.54(5),October 2007,178

[13] L. Buermann, G. Higlers, Significant discrepancies in air kerma rates measured with free-air and cavity ionization chambers, Nucl. Instr.and Meth. A 580(2007), 477-480.

[14] V.I.Razin, A.I.Reshetin, S.N.Filippov, RETGEM with polyvinyl-chloride electrodes, Instruments and Experimental
Techniques, 2011, V.54, №5, pp709-711.

[15] V.I.Razin and A.I.Reshetin, Features of Gas Discharge in Narrow-Gap Micropattern Gas Detectors at High Level of Alpha-Particles Background, Physics of Particles and Nuclei Letters, 2012,V 9, №1,58-61.

[16] V.I.Razin, Study of Streamer Regime for a Narrow-Gap Detector with Simple Gas Infflation, Physics of Particles and Nuclei Letters, 2013, Vol.10, №3,pp.256-257.

[17] C. Shalem, R.Chechik, A.Breskin, K.Michaeli, Advances in Thick GEM-like gaseous electron multipliers-Part1: atmospheric pressure operation, Nucl.Instr.and Meth.A, 558 (2006), 475-489.

[18] R. Chechik, A.Breskin, C.Shalem, D.Mormann, Thick GEM like hole multipliers: properties and possible applications, Nucl.Instr.and Meth.A, 535 (2004),303-308.

[19] V.I.Razin(2014), Metal Gas Electron Multiplier, Universal Journal of Physics and Application, 2,321-324. 